\title{
A LEMMA IN THE THEORY OF STRUCTURAL STABILITY OF DIFFERENTIAL EQUATIONS ${ }^{1}$
}

\author{
PHILIP HARTMAN
}

1. Introduction. Consider the system of real, autonomous, nonlinear differential equations

$$
x^{\prime}=\Gamma x+F(x), \text { where } F(x)=o(|x|) \quad \text { as } x \rightarrow 0,
$$

where $x$ is a vector, $|x|$ the Euclidean length of $x, F(x)$ a smooth vector valued function for small $|x|$, and $\Gamma$ a constant matrix with eigenvalues $\gamma_{1}, \gamma_{2}, \cdots$ satisfying

$$
\operatorname{Re} \gamma_{j} \neq 0 .
$$

M. M. Peixoto has raised the question as to whether or not there exists a topological mapping

$$
R: u=u(x)
$$

of a vicinity of $x=0$ onto a viciniy of $u=0$ such that solution paths of (1.1) are mapped onto solution paths of

$$
u^{\prime}=\Gamma u .
$$

The object of this note is to answer this question in the affirmative when $F(x)$ is of class $C^{2}$. (It can be mentioned that, even if $F(x)$ is analytic, there need not exist such a map $R$ of class $C^{1}$ with nonvanishing Jacobian; [2].) The mapping (1.3) to be obtained below maps solution paths of (1.1) into those of (1.4) preserving parametrizations.

The first part of the paper concerns the linearization of a local homeomorphism of a Euclidean space into another of the same dimension. The second part concerns (1.1) or rather the "group" of homeomorphisms associated with (1.4). The reduction of the problem to mappings is suggested by Sternberg [3].

2. Local homeomorphisms. Let $u, u^{1}, x, x^{1}, X$ be (real) Euclidean $\mu$-vectors and $v, v^{1}, y, y^{1}, Y$ Euclidean $\nu$-vectors. The first theorem to be proved is the following:

Received by the editors October 21, 1959.

1 This research was supported by the United States Air Force through the Air Force Office of Scientific Research of the Air Research and Development Command, under contract No. AF 18(603)-41. Reproduction in whole or in part is permitted for any purpose of the United States Government. 
(I) Let $T:(x, y) \rightarrow\left(x^{1}, y^{1}\right)$ be a map of the form

$$
T: x^{1}=A x+X(x, y), \quad y^{1}=B y+Y(x, y),
$$

where $X, Y$ are of class $C^{1}$,

$$
X, Y=o(|x|+|y|) \text { as }(x, y) \rightarrow 0,
$$

$A$ and $B$ are constant matrices with eigenvalues $a_{1}, \cdots, a_{\mu}$ and $b_{1}, \cdots, b_{\nu}$, respectively, satisfying

$$
0<\left|a_{m}\right|<1<\left|b_{n}\right| \text { for } 1 \leqq m \leqq \mu, 1 \leqq n \leqq \nu .
$$

Then there exists a continuous, one-to-one map

$$
R: u=U(x, y), \quad v=V(x, y)
$$

of a neighborhood of $(x, y)=0$ onto a neighborhood of $(u, v)=0$ such that

$$
R T R^{-1}=L
$$

where $L$ is the linear map

$$
L: u^{1}=A u, \quad v^{1}=B v .
$$

The case where $T$ is a contraction $T: x \rightarrow x^{1}=A x+X(x)$ is simpler than that of (2.1). Actually, (I) implies a corresponding result for such a map since $T$ can be extended by letting $y^{1}=B y$.

The proof of (I) will be by the method of successive approximations and similar to the proof of (IV) in [2].

3. Proof of (I). The map $R$. After separate linear changes of the $x$ and $y$ variables, it can be supposed that

$$
|A|<a<1, \quad\left|B^{-1}\right|<1 / b<1,
$$

where $|A|$ is the norm: sup $|A x|$ for $|x|=1$, and $a, b$ are constants.

Theorem (III) of [2] shows that there is no loss of generality in assuming that the planes $x=0$ and $y=0$ are invariant, that is, that

$$
X(0, y) \equiv Y(x, 0) \equiv 0 .
$$

Hence, there is a constant $K$ satisfying

$$
|X(x, y)| \leqq K|x|, \quad|Y(x, y)| \leqq K|y| .
$$

Let $r>0$ be an arbitrarily small, fixed, positive number. It can be supposed that $T$ is a $C^{1}$, one-to-one map of the entire $(x, y)$-space onto the $\left(x^{1}, y^{1}\right)$-space and that

$$
X(x, y) \equiv Y(x, y) \equiv 0 \text { if }|x|^{2}+|y|^{2} \geqq r^{2} .
$$


For, in (2.1), $X$ and $Y$ can be replaced by $X(x, y) \phi\left(|x|^{2}+|y|^{2}\right)$ and $Y(x, y) \phi\left(|x|^{2}+|y|^{2}\right)$, respectively, where $\phi=\phi(t)$ is a $C^{1}$ function of a real variable $t, 0 \leqq t<\infty$, satisfying $\phi(t) \equiv 1$ for $0 \leqq t \leqq r / 2, \phi(t) \equiv 0$ for $t \geqq r$ and $|d \phi / d t| \leqq 3 / r$. If $|x|^{2}+|y|^{2} \leqq r^{2}$, then $\partial\left\{X(x, y) \phi\left(|x|^{2}+|y|^{2}\right)\right\} / \partial x^{i}=\phi \partial X / \partial x^{i}+2 x^{i} X d \phi / d t$ is majorized by $\left|\partial X / \partial x^{i}\right|+6|X|$, which has an arbitrarily small bound if $r>0$ is sufficiently small. Since the same is true for all partial derivatives of $X \phi$ and $Y \phi$, it is clear that the mapping $T$ obtained by replacing $X, Y$ by $X \phi, Y \phi$ has the desired properties.

Note that the constant $K$ in (3.3) can be chosen arbitrarily small if $r>0$ in (3.4) is sufficiently small. Hence it can be supposed that

$$
\left|x^{1}\right| \leqq a|x|, \quad\left|y^{1}\right| \geqq b|y| .
$$

Let the inverse of (2.1) be

$$
T^{-1}: x=A^{-1} x^{1}+X_{1}\left(x^{1}, y^{1}\right), \quad y=B^{-1} y+Y_{1}\left(x^{1}, y^{1}\right)
$$

so that, by virtue of (2.1),

$$
X(x, y)=-A X_{1}\left(x^{1}, y^{1}\right), \quad Y(x, y)=-B Y_{1}\left(x^{1}, y^{1}\right) .
$$

The functions $X_{1}, Y_{1}$ satisfy the analogues of (2.2), (3.2), (3.3) and (3.4).

Let the desired relation (2.5) be written as

$$
L R=R T \text {. }
$$

This is equivalent to the functional equation for $V$ given by

$$
B V(x, y)=V(A x+X(x, y), B y+Y(x, y))
$$

and an analogous one for $U$ which, in terms of $\left(x^{1}, y^{1}\right)$ variables, is

$$
U\left(x^{1}, y^{1}\right)=A U\left(A^{-1} x^{1}+X_{1}\left(x^{1}, y^{1}\right), B^{-1} y^{1}+Y_{1}\left(x^{1}, y^{1}\right)\right) .
$$

A solution $V(x, y)$ of (3.9) will be obtained by successive approximations. Put

(3.110) $\quad V_{0}(x, y)=y$,

$\left(3.11_{n}\right) \quad V_{n}(x, y)=B^{-1} V_{n-1}(A x+X(x, y), B y+Y(x, y))$

for $n=1,2, \cdots$. Let

$$
V^{n}(x, y)=V_{n}(x, y)-V_{n-1}(x, y) \quad \text { for } n=1,2, \cdots,
$$

so that, for $n=2,3, \cdots$,

$$
V^{n}(x, y)=B^{-1} V^{n-1}(A x+X(x, y), B y+V(x, y)) .
$$

By $\left(3.11_{0}\right)$ and $\left(3.11_{1}\right)$, it follows that 


$$
V^{1}(x, y)=B^{-1} Y(x, y) .
$$

It will be shown by induction that there exist positive numbers $\theta(<1), \delta(<1), K_{1}$ such that

$$
\left|V^{n}(x, y)\right| \leqq K_{1} \theta^{n}|y|^{\delta}
$$

for $n=1,2, \cdots$. That such an inequality is valid for $n=1$ follows from (3.3), (3.4) and (3.14). Suppose that (3.15) holds for a given $n \geqq 1$. By (3.1) and (3.13), $\left|V^{n+1}(x, y)\right| \leqq b^{-1} K_{1} \theta^{n}|B y+Y|^{8}$. It is clear from (3.3) that there exists a constant $b_{1}>0$ such that $|B y+Y|$ $\leqq b_{1}|y|$. Let $\delta>0$ be so small that $\theta=b^{-1} b_{1}^{\delta}<1$. Then $\left|V^{n+1}(x, y)\right|$ $\leqq K_{1} \theta^{n+1}|y|^{\delta}$. This completes the verification of (3.15) for $n=1,2, \cdots$.

It follows from (3.15) that $V_{0}, V_{1}, \cdots$ converges (uniformly on bounded $(x, y)$-sets) to a continuous function $V$ satisfying (3.9). Similarly the successive approximations

(3.160) $\quad U_{0}\left(x^{1}, y^{1}\right)=x^{1}$,

(3.16n) $U_{n}\left(x^{1}, y^{1}\right)=A U_{n-1}\left(A^{-1} x^{1}+X_{1}\left(x^{1}, y^{1}\right), B^{-1} y^{1}+Y_{1}\left(x^{1}, y^{1}\right)\right)$

for $n=1,2 \cdots$ converge to a continuous solution $U$ of (3.10). The functions $U(x, y), V(x, y)$ define a continuous map $R$, for all $(x, y)$ satisfying (3.8).

4. Proof of (I) completed. It remains to show that $R$ is one-to-one (in which case, (2.5) is a consequence of (3.8)). The proof that $R$ is one-to-one, if $r>0$ is sufficiently small, will depend on

$$
\begin{aligned}
& U(x, y)=x \text { if }|x| \geqq r / a \text { and } V(x, y)=y \text { if }|y| \geqq r, \\
& U(x, y)=0 \text { if } x=0 \text { and } V(x, y)=0 \text { if } y=0 .
\end{aligned}
$$

These relations follow from the definitions (3.11) and (3.16) in view of (3.2), (3.4) and (3.5).

Suppose, if possible, that $R$ is not one-to-one, so that there exists a pair of points $\left(x_{1}, y_{1}\right),\left(x_{2}, y_{2}\right)$ such that

$$
R\left(x_{1}, y_{1}\right)=R\left(x_{2}, y_{2}\right) \text { but }\left(x_{1}, y_{1}\right) \neq\left(x_{2}, y_{2}\right) .
$$

It follows from (3.8) and a simple induction that

$$
R\left(x_{1}^{n}, y_{1}^{n}\right)=R\left(x_{2}^{n}, y_{2}^{n}\right) \quad \text { for } n=0, \pm 1, \cdots,
$$

where $\left(x_{j}^{n}, y_{j}^{n}\right)=T^{n}\left(x_{j}, y_{j}\right)$. Since $T$ is one-to-one,

$$
\left(x_{1}^{n}, y_{1}^{n}\right) \neq\left(x_{2}^{n}, y_{2}^{n}\right) \quad \text { for } n=0,1, \cdots .
$$

Suppose that 


$$
\left|x_{1}\right|,\left|y_{1}\right|,\left|x_{2}\right|,\left|y_{2}\right| \neq 0 .
$$

It will be clear from the arguments to follow that if one of these inequalities fails to hold, then (4.4) implies $x_{1}=x_{2}=0, y_{1}=y_{2}=0$.

In view of (4.6) and (3.5), it can be supposed that $\left|x_{1}\right| \geqq r / a$, $\left|x_{2}\right| \geqq r / a$ (for otherwise $\left(x_{j}, y_{j}\right)$ can be replaced by $\left(x_{j}^{-k}, y_{j}^{-k}\right)$ where $k$ is a large, fixed positive integer). Hence (4.1) and (4.3) show that

$$
x_{1}=x_{2} \text { and } y_{1} \neq y_{2} \text {. }
$$

For $n=0,1, \cdots$, put

$$
s^{n}=\left|x_{2}^{n}-x_{1}^{n}\right|, \quad t^{n}=\left|y_{2}^{n}-y_{1}^{n}\right| ;
$$

so that, by (4.5) and (4.7),

$$
s^{0}=0, \quad t^{0} \neq 0 \text { and } s^{n}+t^{n}>0 .
$$

It will be shown that if $r>0$ in (3.4) had been chosen sufficiently small, then, for $n=0,1, \cdots$,

$$
0 \leqq s^{n} \leqq t^{n} .
$$

The relation (3.4) and the argument following it show that the partial derivatives of $X, Y$ have arbitrarily small bounds if $r>0$ is sufficiently small. Suppose, therefore, that

$$
\begin{gathered}
\left|X(x, y)-X\left(x^{*}, y^{*}\right)\right|,\left|Y(x, y)-Y\left(x^{*}, y^{*}\right)\right| \\
\leqq \epsilon\left(\left|x-x^{*}\right|+\left|y-y^{*}\right|\right)
\end{gathered}
$$

for all $(x, y),\left(x^{*}, y^{*}\right)$ and that $\epsilon>0$ is so small that $a+2 \epsilon<1$ and $b-2 \epsilon>1$. If (4.10) holds for some fixed $n$, then

$$
\begin{aligned}
& s^{n+1} \leqq a s^{n}+\epsilon S^{n}+\epsilon t^{n} \leqq(a+2 \epsilon) t^{n} \leqq t^{n}, \\
& t^{n+1} \geqq b t^{n}-\epsilon S^{n}-\epsilon t^{n} \geqq(b-2 \epsilon) t^{n} \geqq t^{n},
\end{aligned}
$$

so that $s^{n+1} \leqq t^{n+1}$. Hence (4.10) holds for $n=0,1, \cdots$.

In view of (4.6) and (3.5), $\left|y_{1}^{n}\right| \geqq r$ and $\left|y_{2}^{n}\right| \geqq r$ for large $n$. But then (4.1) and (4.4) show that $y_{1}^{n}=y_{2}^{n}$, that is, $t^{n}=0$. By (4.10), $s^{n}=t^{n}=0$. But this contradicts the last part of (4.9). Consequently, (4.3) cannot hold and (I) is proved.

5. Differential equations. In (1.1), let $F(x)$ be of class $C^{1}$. Let the general solution of (1.1) passing through $(x, y)$ at $x=0$ be given by $x(t)=\xi(t, x)$. Then $\xi(t, x)$ is of class $C^{1}$ (since $F$ is) and

$$
\xi(t, x)=e^{\Gamma t} x+X(t, x), \text { where } \quad X=o(|x|) \quad \text { as } x \rightarrow 0,
$$

(for $t$ fixed). Consider the set of transformations 


$$
T^{t}: x^{t}=\xi(t, x)=e^{\Gamma t}+X(t, x) .
$$

They satisfy

$$
T^{s} T^{t}=T^{s+t} \text { for small }|x| .
$$

(II) In the system of differential equations (1.1), let $F(x)$ be of class $C^{2}$ for small $|x|$ and let the eigenvalues of $\Gamma$ satisfy (1.2). Let $x(t)$ $=\xi(t, x)$ be the solution of (1.1) satisfying $x(0)=x$. Then there exists a continuous, one-to-one map (1.3) of a neighborhood of $x=0$ onto a neighborhood of $u=0$ such that $R T^{t} R^{-1}$ is the linear map

$$
L^{t}: u^{t}=e^{\Gamma t} u .
$$

By Theorem (I) just proved, there is a topological map $R_{1}: x \rightarrow u$ of a vicinity of $x=0$ onto a vicinity of $u=0$ such that $S^{t}=R_{1} T^{t} R_{1}^{-1}$ satisfies $S^{1}=L^{1}$ (hence, $S^{n}=L^{n}$ for $\left.n=0, \pm 1, \cdots\right)$ ). According to an observation of Sternberg [3, p. 817], the continuous map

$$
R_{2}=\int_{0}^{1} L^{-t} S^{t} d t
$$

then satisfies $L^{t} R_{2}=R_{2} S^{t}$, so that $L^{t} R=R T^{t}$ if $R=R_{2} R_{1}$. Thus (II) would be proved (for $F(x)$ of class $C^{1}$ ) if it could be verified that $R_{2}$ is one-to-one (for small $|x|$ ). Since I have not been able to give a verification of this, the proof of (II) below will involve a detour. This detour necessitates the assumption that $F(x)$ is of class $C^{2}$, rather than of class $C^{1}$.

The role of the assumption that $F(x)$ is of class $C^{2}$ is to assure the existence of a change of variables of class $C^{1}$ transforming (1.1) into a new system which is linear on each of the invariant manifolds consisting of solution paths reaching the origin for $t= \pm \infty$. This is used to obtain the relation $S^{t}=L^{t}$ on these invariant manifolds.

It will be verified in $\S 8$ that (II) remains correct if $F(x)$ is only assumed of class $C^{1}$ if the real parts of the eigenvalues $\gamma_{j}$ of $\Gamma$ are of the same sign, say

$$
\operatorname{Re} \gamma_{j}<0 .
$$

It will remain undecided whether or not this is the case without the assumption (5.6).

6. Proof of (II). Preliminary changes of variables. It will always be assumed that the vectors $u, v, x, y, \cdots$ occurring below are small in magnitude.

It will be supposed that the real parts of the eigenvalues of $\Gamma$ are not all of the same sign. (The proof for the other case is implicit in 
the proof to be given; cf. also $\S 8$ below.)

After a linear change of variables and a change of notation, it can be supposed that the system of differential equations to be considered is of the form

$$
x^{\prime}=\Gamma x+F(x, y), \quad y^{\prime}=\Delta y+G(x, y),
$$

where $x$ is a $\mu$-vector, $y$ a $\nu$-vector, $\Gamma$ and $\Delta$ constant matrices with eigenvalues $\gamma_{j}, \delta_{k}$ satisfying

$$
\operatorname{Re} \gamma_{j}<0, \quad \operatorname{Re} \delta_{k}>0,
$$

respectively, $F$ and $G$ are of class $C^{2}$ for small $|x|,|y|$ and

$$
F, G=o(|x|+|y|) \text { as }|x|+|y| \rightarrow 0 .
$$

It can also be supposed that there exists a constant $c>0$ such that

$$
x \cdot \Gamma x \leqq-c|x|^{2}, \quad y \cdot \Delta y \geqq c|y|^{2},
$$

where the dot denotes scalar multiplication.

Let the analogue of (5.2), in the present notation, be

$$
T^{t}: x^{t}=e^{\Gamma t} x+X(t, x, y), \quad y^{t}=e^{\Delta t} y+Y(t, x, y) ;
$$

so that $x(t)=x^{t}, y(t)=y^{t}$ is the solution of (6.1) determined by the initial condition $x(0)=x, y(0)=y$. The functions $X, Y$ are of class $C^{2}$ and satisfy $X, Y=o(|x|+|y|)$ as $|x|+|y| \rightarrow 0$ for fixed $t$.

After a $C^{2}$ change of variables, which leaves the origin fixed and which has a Jacobian matrix reducing to the unit matrix at the origin, it can be supposed that $F(0, y)=G(x, 0)=0$. Thus the planes $x=0$ and $y=0$ are invariant, so that $X(t, 0, y) \equiv Y(t, x, 0) \equiv 0$. (The existence of such a change of variables of class $C^{1}$, when $F, G$ is of class $C^{1}$, follows from [1, p. 333]. This result can be easily extended to the desired one. The desired result is implied directly by Theorem (III) of [2] since the set of points $(x, y)$ satisfying $T^{t}(x, y) \rightarrow(0,0)$ as $t \rightarrow \infty$ or $t \rightarrow-\infty$ is not changed if the continuous variable $t$ is replaced by a discrete variable $n=0, \pm 1, \cdots$.)

According to Theorem (II) of [2], there exists a $C^{1}$ change of variables, say $R_{1}$, with the properties that it leaves the planes $x=0$ and $y=0$ fixed, it has a Jacobian matrix reducing to the unit matrix at the origin, and the map $S^{t}=R_{1} T^{t} R_{1}^{-1}$ satisfies $S^{1}=L^{1}$ on the invariant manifolds $x=0$ and $y=0$.

Let $R_{2}$ be defined by (5.5). Then (5.5) is of class $C^{1}$ with a Jacobian matrix reducing to the unit matrix at the origin. It is readily seen that $L^{t} R_{2}=R_{2} S^{t}$ on the invariant manifolds $x=0$ and $y=0$; cf. [3, p. 817]. Put $R_{0}=R_{2} R_{1}$, so that $R_{0} T^{t} R_{0}^{-1}=L^{t}$ on $x=0$ and on $y=0$. 
Make the change of variables $(x, y) \rightarrow R_{0}(x, y)$. Let the new variables be called $(x, y)$ again, let $(6.1)$ be the new set of differential equations and let $R_{0} T^{t} R_{0}^{-1}$ be renamed $T^{t}$.

The new functions $F, G$ have all of the properties mentioned above except that they are only continuous (instead of class $C^{2}$ ) and, in addition,

$$
F=G=0 \text { if }|x||y|=0 .
$$

The new functions $X, Y$ have all of the properties mentioned above except that they are only of class $C^{1}$ (instead of class $C^{2}$ ) and, in addition,

$$
X=Y=0 \text { if }|x||y|=0 .
$$

7. Proof of (II) completed. By Theorem (I) and its proof above, there exists a topological map

$$
Q: x=f(u, v), \quad y=g(u, v)
$$

of a vicinity of $(u, v)=(0,0)$ onto a vicinity of $(x, y)=(0,0)$ such that

$$
Q^{-1} T^{1} Q=L^{1}: u^{1}=e^{\Gamma} u, v^{1}=e^{\Delta} v
$$

and

$$
Q(u, v) \equiv(f(u, v), g(u, v))=(u, v) \quad \text { if }|u||v|=0 .
$$

(The map $Q$ is the inverse of the map $R$ in (I).)

Consider the linear system of differential equations

$$
u^{\prime}=\Gamma u, \quad v^{\prime}=\Delta v .
$$

By (6.4),

$$
u \cdot u^{\prime} \leqq-c|u|^{2}, \quad v \cdot v^{\prime} \geqq c|v|^{2} ;
$$

so that $|u|^{2}$ is decreasing and $|v|^{2}$ is increasing along every solution path not in the planes $u=0$ and $v=0$.

The solution of (7.4) beginning at $(u, v)$ for $t=0$ is given by $u=u^{t}$, $v=v^{t}$, where

$$
L^{t}: u^{t}=e^{\Gamma t} u, \quad v^{t}=e^{\Delta t} .
$$

If $|u||v| \neq 0$, there is a unique $t$-value, say $t=\alpha(u, v)$, such that $\mid \begin{aligned} & u^{t}|=| v^{t} \mid \text { if } t=\alpha \text {. The function } \alpha(u, v) \text { is continuous on the set } \\ & u|| v \mid \neq 0 \text {. }\end{aligned}$ Define a map $(u, v) \rightarrow(x, y)$ as follows:

$$
P: \begin{array}{ll}
(x, y)=(u, v) & \text { if }|u||v|=0, \\
(x, y)=T^{-\alpha} Q L^{\alpha}(u, v) & \text { if }|u||v| \neq 0,
\end{array}
$$


where $\alpha=\alpha(u, v)$. It is clear that (7.7) is defined for small $|u|,|v|$ and is continuous for $|u||v| \neq 0$.

In order to clarify the meaning of (7.7), note that the first part means that $P$ is the identity map on the planes $u=0$ and $v=0$. Since (7.2) implies that $Q^{-1} T^{n} Q \equiv L^{n}$ for $n=0, \pm 1, \cdots$, the last part of (7.7) means that $P=Q$ on the cone $C:|u|=|v|$ and its iterates $L^{n} C$ for $n=0, \pm 1, \cdots$, where $\alpha=-n$.

It will first be verified that

$$
T^{-t} P L^{t}(u, v)=P(u, v) .
$$

This is clear if $|u||v|=0$ from (7.3), the first part of (7.7), and the fact that $T^{t}=L^{t}$ on the planes $u=0$ and $v=0$. If $|u||v| \neq 0$, then $\alpha\left(L^{t}(u, v)\right)=\alpha(u, v)-t$, so that $P L^{t}(u, v)=\left\{T^{-(\alpha-t)} Q L^{\alpha-t}\right\} L^{t}(u, v)$. Thus (7.8) follows.

The map $P$ is one-to-one. In order to see this, it is sufficient to examine the action of $P$ on $|u||v| \neq 0$. It is clear from (7.8) that solution paths of (7.4) are mapped onto solution paths of (6.1). The last part of (7.7) shows that $P$ is one-to-one on any given solution path of (7.4). Also, $P$ sends different solution paths of (7.4) into different solution paths of (6.1) since different solution paths of (7.4) meet $C:|u|=|v|$ in different points and $P=Q$ is one-to-one on $C$.

Finally, it has to be verified that $P$ is continuous at points where $u=0$ or $v=0$. In order to see this, note that

$$
P(u, v)=T^{-\alpha+n} Q L^{\alpha-n}(u, v)
$$

for any integer $n$. Let $n=n(u, v)$ be chosen so that $0 \leqq \alpha-n<1$. If $(u, v)$ is near a plane $u=0$ or $v=0$, then $L^{t}(u, v)$ remains near the corresponding plane for $0 \leqq t \leqq 1$. Also, $Q$ is nearly the identity mapping near these planes. Finally, $T^{-t}$ for $0 \leqq t \leqq 1$ is approximately $L^{-t}$ near these planes (since $T^{-t}=L^{-t}$ on these planes). It follows that $P$ is nearly the identity map near the planes $u=0$ and $v=0$. Since $P$ is the identity map on $u=0$ and $v=0$, the continuity of $P$ follows.

Thus, $R=P^{-1}$ has the asserted properties and the proof of (II) is complete.

8. Remark. If (5.6) holds, the proof of (II) can be given more simply even if it is only assumed that $F(x)$ in (1.1) is only of class $C^{1}$. After a linear change of variables, it can be supposed that

$$
x \cdot \Gamma x \leqq-c|x|^{2} \leqq 0 .
$$

To every $u$ satisfying $0<|u| \leqq r$, there exists a unique number $\alpha=\alpha(u) \leqq 0$ such that $u^{t}$ defined by

$$
L^{t}: u^{t}=e^{\Gamma t} u
$$


satisfies $\left|u^{t}\right|=r$ if $t=\alpha$. Let a map $u \rightarrow x$ be defined by

$$
P: \begin{array}{ll}
x=0 & \text { if } u=0, \\
x=T^{-\alpha} L^{\alpha} u & \text { if } u \neq 0 .
\end{array}
$$

It can then be verified that $R=P^{-1}$ exists and has the desired properties.

9. The smoothness of $R$ in (I). It is easy to see from the proof of (I) that the map (2.4) is of class $C^{1}$ at any point not on the invariant manifolds. (It is not clear whether or not $R^{-1}$ has the same property.) The following example (cf. [2, Part IV]) shows that there need not exist a linearizing map $R$ which is of class $C^{1}$ everywhere except at the origin.

The system of three scalar differential equations

$$
x^{\prime}=\alpha x, \quad y^{\prime}=(\alpha-\gamma) y+\epsilon x z, \quad z^{\prime}=-\gamma z,
$$

where $\alpha>\gamma>0$ and $\epsilon \neq 0$, has the general solution

$$
T^{t}: x^{t}=x e^{\alpha t}, \quad y^{t}=(y+\epsilon x z t) e^{(\alpha-\gamma) t}, \quad z^{t}=z e^{-\gamma t} .
$$

Consider the analytic map $T=T^{1}$,

$$
T: x^{1}=a x, \quad y^{1}=a c(y+\epsilon x z), \quad z^{1}=c z,
$$

where $a=e^{\alpha}$ and $c=e^{-\gamma}$, so that $a>1>c>0$ and $a c>1$. It is readily verified that each of the maps

$$
R: u=x, \quad v=y-\epsilon x z \log |x| / \log a, \quad w=z,
$$

and

$$
R: u=x, \quad v=y-\epsilon x z \log |z| / \log c, \quad w=z,
$$

linearizes (9.3), in fact, (9.2) for all $t$. It turns out that the nonexistence of some derivatives of these maps at $x=0$ or $z=0$ cannot be avoided.

It will be shown that if

$$
R^{-1}: x=f(u, v, w), \quad y=g(u, v, w), \quad z=h(u, v, w)
$$

is a local homeomorphism linearizing (9.3), then either the partial derivative $g_{u}(0,0, w)$ fails to exist for all $w \neq 0$ or $g_{w}(u, 0,0)$ fails to exist for all $u \neq 0$.

The considerations below concern only $v=0$ (and small $|u|,|w|$ ). Write $j(u, w)$ for $j(u, 0, w)$ if $j=f, g, h$. The relation (2.5) in the form $T R^{-1}=R^{-1} L$ gives, for $v=0$,

$$
a f=f(a u, c w), \quad a c[g+\epsilon f h]=g(a u, c w), \quad c h=h(a u, c w),
$$


where the argument of $f, g, h$ on the left is $(u, w)$. A simple induction shows that $a^{n} f=f\left(a^{n} u, c^{n} w\right)$,

$$
a^{n} c^{n}[g+n \in f h]=g\left(a^{n} u, c^{n} w\right),
$$

and $c^{n} h=h\left(a^{n} u, c^{n} w\right)$ for small $|u|,|w|$. The first of these relations implies that $f(0, w)=a^{-n} f\left(0, c^{n} w\right) \rightarrow 0$ as $n \rightarrow \infty$. Hence $f(0, w) \equiv 0$. Similarly, $h(u, 0) \equiv 0$. It then follows, from $a c>1$, that $g(0, w) \equiv 0$.

Multiply (9.5) by $c^{-n}$ and replace $u$ by $u / a^{n}$ to obtain

$$
a^{n} g\left(u / a^{n}, w\right)+n \in f\left(u, c^{n} w\right) h\left(u / a^{n}, w\right)=c^{-n} g\left(u, c^{n} w\right) .
$$

Suppose that $g_{u}(0, w), g_{w}(u, 0)$ exist (for a fixed $u$ and $w$ ), then $a^{n} g\left(u / a^{n}, w\right)=a^{n}\left[g\left(u / a^{n}, w\right)-g(0, w)\right]=O(1)$ and $c^{-n} g\left(u, c^{n} w\right)$ $=c^{-n}\left[g\left(u, c^{n} w\right)-g(u, 0)\right]+c^{-n} g(u, 0)=O(1)+c^{-n} g(u, 0)$ as $n \rightarrow \infty$. Thus (9.6) shows that $c^{-n} g(u, 0)=O(n)$. Since $c<1$, it follows that $g(u, 0)=0$. But then (9.6) implies that $f(u, 0) h(0, w)=0$, which is impossible for $u w \neq 0$. For example, if $f(u, 0)=0$ and $u \neq 0$, then $f=g=h=0$ when $(u, v, w)$ is the point $(u, 0,0) \neq(0,0,0)$. This contradicts the fact that $R^{-1}$ is one-to-one. It follows that if $g_{u}(0, w)$ exists for some $w \neq 0$, then $g_{w}(u, 0)$ does not exist for any $u \neq 0$.

\section{REFERENCES}

1. E. A. Coddington and N. Levinson, Theory of ordinary differential equations, New York, 1955.

2. P. Hartman, On local homeomorphisms of Euclidean spaces, Proceedings of the Symposium on Ordinary Differential Equations, Mexico City, 1959.

3. S. Sternberg, Local contractions and a theorem of Poincare, Amer. J. Math. vol. 79 (1957) pp. 809-824.

The Johns Hopkins UnIversity 